\title{
LIMIT PROPERTIES OF POISSON KERNELS OF TUBE DOMAINS
}

\author{
BY
}

\section{LAWRENCE J. DICKSON (1)}

\begin{abstract}
If certain local boundary conditions hold near $P \in \partial \Gamma$, the Poisson kernel belonging to a proper cone $\Gamma \subset \mathbf{R}^{n}$ converges to a tight $C_{0}^{*}$ limit as its parameter converges admissibly to $P$ in $\Gamma$. This limit can be identified with a lower-dimensional Poisson kernel. The result always works for polytopic and "rounded" cones; for these, a result on the decrease at infinity is obtained which in fact implies convergence almost every where in the appropriate sense of the Poisson integral to certain of its boundary values.
\end{abstract}

1. Preliminaries and notation.

A. Some generalities. Tube domains of $\mathrm{C}^{n}$ are domains of holomorphy of the form $\Omega=\Omega_{\Gamma}=\Gamma \oplus i \mathbf{R}^{n}$, where $\Gamma$ is a proper cone of $\mathbf{R}^{n}$. By "cone" we mean any set $\Delta$ such that $\epsilon \Delta=\Delta \forall \epsilon>0$; a proper cone of a vector space $T$ over the reals is a nonempty, open (in the topology of $T$ ) convex cone whose closure contains no whole line. Here, as throughout this paper, all vector spaces are assumed to be subspaces of $\mathbf{R}^{n}$ (or $\mathrm{C}^{n}$ ) with the induced inner product for some nonnegative integer $n$. All direct sums are orthogonal, and vector space identifications $(\approx)$ are unitary. The letter $\Gamma$, with or without subscripts, always denotes a proper cone.

The distinguished boundary of a tube domain is the set $i \mathbf{R}^{n} \cdot H^{2}(\Omega)$ has a reproducing kernel, the Szegö kernel, which may be represented as

$$
K_{\mathbf{\Gamma}}(X+i Y)=K_{\mathbf{\Gamma} ; X}(Y)=\int_{\Gamma^{*}} e^{-2 \pi(X+i Y, V)} d V, \quad X \in \Gamma, Y \in \mathbf{R}^{n},
$$

where $\Gamma^{*}=\left\{Y \in \mathbf{R}^{n}:(X, Y)>0 \forall X \in \bar{\Gamma}-\{0\}\right\}$ is the dual cone of $\Gamma$, also a proper cone. The reproducing property is expressed by

$$
F(X+i Y)=\left(f * K_{\mathbf{r} ; X}\right)(Y),
$$

where $F \in H^{2}(\Omega)$ and $f(Y)=F(i Y)$ is its limit at the distinguished boundary.

Received by the editors August 3, 1972 and, in revised form, November 11, 1972. $32 \mathrm{~F} 05$.

AMS (MOS) subject classifications (1970). Primary 31B10, 52A20; Secondary 31B25,

Key words and phrases. Szegö and Poisson kernels, proper cones, tight $C_{0}^{*}$ limit, limit cones, maximal operator, decrease at infinity.

(1) This research was partially supported by the National Science Foundation. 
The fact that $K$ commutes with the conjugate operator leads to the definition of another kernel, the Poisson kernel,

$$
P_{\mathbf{r}}(X, Y)=P_{\mathbf{r} ; X}(Y)=\left\|K_{\mathbf{r} ; X}\right\|_{2}^{-2}\left|K_{\mathbf{S} ; X}(Y)\right|^{2},
$$

which is positive, $C_{0}$, has unit integral, and satisfies the reproducing property

$$
F(X+i Y)=\left(f * P_{\Gamma ; X}\right)(Y)=P f_{\Gamma}(X, Y)
$$

for wide classes of holomorphic functions $F$, including all the $H^{p}$ 's. For any suitable $f$, the value of the Poisson integral $P f$ at each point is thus a weighted average of the "boundary" values $f$. In this paper we investigate the shape of the weighting function $P_{\Gamma ; X}(\S 3 A)$, and its behavior as $X$ nears 0 or other points of $\partial \Gamma$. The limits we find are identified with lower-dimensional Poisson kernels. We reach some conclusions about the boundary behavior of $P f$.

For $X \in \Gamma$ we define the compact section $C_{\Gamma^{*} ; X}=C_{X}^{*}$ of $\bar{\Gamma}^{*}$ as follows:

$$
C_{X}^{*}=\left\{Y \in \bar{\Gamma}^{*}:(X, Y)=1\right\}
$$

and similarly for $C_{r ; Y}=C_{Y}, Y \in \bar{\Gamma}^{*}$. These are always nonempty and compact.

Suppose $A: \mathbf{R}^{n} \rightarrow \mathbf{R}^{n}$ is a nonsingular linear transformation. We will denote by $A^{\#}$ the transformation $A^{*-i}=\left(A^{-1}\right)^{*}$. We note $\cdot(A \Gamma)^{*}=A^{\#} \Gamma^{*}$. The following claims of $\S 1 \mathrm{~A}$ are simple algebraic consequences of our definitions.

1.1 Claim. If $\hat{f}(V)$ is the Fourier transform of $f(Y)$, then

$$
\widehat{K_{\Gamma ; X}}(V)=\chi_{\Gamma^{*}}(V) e^{-2 \pi(X, V)}
$$

$$
\widehat{P_{\Gamma ; X}}(V)=\left\|K_{\Gamma ; X}\right\|_{2}^{-2} \int_{H\left(\Gamma^{*}, V\right)} e^{-4 \pi(X, Y)} d y,
$$

$$
\text { where } H\left(\Gamma^{*}, V\right)=\left(\Gamma^{*}+V / 2\right) \cap\left(\Gamma^{*}-V / 2\right)
$$

1.2 Claim. For $A: \mathbf{R}^{n} \rightarrow \mathbf{R}^{n}$ nonsingular, $Z=X+i Y$, the following bold:

$$
\begin{aligned}
& K_{A \Gamma}(A Z)=|\operatorname{det} A|^{-1} K_{\Gamma}(Z) ; \\
& P_{A \Gamma ; A X}(A Y)=|\operatorname{det} A|^{-1} P_{\Gamma ; X}(Y) \text {; } \\
& \widehat{K_{A \Gamma ; A X}}\left(A^{\#} V\right)=\widehat{K_{\Gamma ; X}}(V) \text {; } \\
& \widehat{P}_{A \Gamma ; A X}\left(A^{\#} V\right)=\overbrace{\Gamma ; X}(V) \text {. }
\end{aligned}
$$


1.3 Definition. For $f_{i}: D_{i} \rightarrow \mathrm{C}$, we define the direct product $f_{1} \times f_{2}$ on $D_{1} \oplus D_{2}$ as follows:

$$
\left(f_{1} \times f_{2}\right)\left(x_{1}+X_{2}\right)=f_{1}\left(x_{1}\right) f_{2}\left(X_{2}\right), \quad X_{i} \in D_{i} .
$$

1.4 Claim. If $\Gamma_{i}$ of $\mathbf{R}^{l_{i}}$ are proper cones then so is $\Gamma_{1} \oplus \Gamma_{2} \subset \mathbf{R}^{l_{1}+l_{2}}$, and its Szegö and Poisson kernels and their Fourier transforms are direct products (in $(X, Y)$ or $(X, V)$ respectively) of those of the $\Gamma_{i}$.

1.5 Definition. Suppose $\Gamma, \Gamma_{1}, \ldots, \Gamma_{m}$ are proper cones of $\mathbf{R}^{n}$. Then $\Gamma$ is the special intersection of the $\Gamma_{i}$ if the following conditions hold:

$$
\begin{gathered}
\Gamma_{i}^{*} \cap \Gamma_{j}^{*}=\varnothing \text { if } i \neq j ; \\
\bigcup_{i=1, \cdots, m} \bar{\Gamma}_{i}^{*}=\bar{\Gamma}^{*}
\end{gathered}
$$

1.6 Claim. If $\Gamma$ is the special intersection of $\left\{\Gamma_{i}: i=1, \cdots, m\right\}$, then

$$
\Gamma=\bigcap_{i=1}^{m} \Gamma_{i} ;
$$

and, on the domains of $K_{\Gamma}$ and $P_{\Gamma}$, respectively,

$$
\begin{gathered}
K_{\mathbf{\Gamma}}=\sum_{i=1}^{m} K_{\boldsymbol{\Gamma}_{i}}, \\
P_{\mathbf{\Gamma}} \leq m \sum_{i=1}^{m} P_{\boldsymbol{\Gamma}_{i}} .
\end{gathered}
$$

B. Tight $C_{\sigma}^{*}$ convergence and the $L^{1}$ limit.

1.7 Definition. $\lim _{\delta \rightarrow 0} f_{\delta}=\mu$ (tight $C_{0}^{*}$ ) if the following hold:

(a) $\mu \in C_{0}^{*}\left(\mathbf{R}^{n}\right)$, and for some $\delta_{0}>0, f_{\delta} \in L^{1}\left(\mathbf{R}^{n}\right)$, if $|\delta| \leq \delta_{0}$;

(b) for any $g \in C_{0}\left(\mathbf{R}^{n}\right), \lim _{\delta \rightarrow 0} \int g(X) f_{\delta}(X) d X=\int g d \mu$;

(c) $\lim \sup _{\delta \rightarrow 0}\left\|f_{\delta}\right\|_{1}<\infty$;

(d) for every $\epsilon>0, \quad \exists \delta(\epsilon)>0$ and a compact set $K \subset \mathbf{R}^{n}$ such that $|\delta| \leq$ $\delta(\epsilon) \Rightarrow \int_{\mathrm{R}^{n}-K}\left|f_{\delta}\right|<\epsilon$.

1.8 Lemma. If $\lim _{\delta \rightarrow 0} f_{\delta}=\mu$ (tight $C_{0}^{*}$ ) then for any $b \in L^{1}, b * \mu \in L^{1}$ and $\lim _{\delta \rightarrow 0} b * f_{\delta}=b * \mu$ in the $L^{1}$ norm.

Proof. It is well known that $b * \mu$ exists a.e. and is $L^{1}$ (with norm $\left.\leq\|b\|_{1}\|\mu\|\right)$. The proof now proceeds by setting $b=g_{\epsilon}+b_{\epsilon}^{\prime}, g_{\epsilon} \in C_{00},\left\|b_{\epsilon}^{\prime}\right\|_{1} \leq \epsilon$. 
1.9 Claim. (a) Suppose $A: \mathbf{R}^{n} \rightarrow \mathbf{R}^{n}$ is nonsingular, $f_{\delta}^{A}(Y)=f_{\delta}\left(A^{-1} Y\right)$, $\mu^{A}(S)=\mu\left(A^{-1} S\right)$. Then $f_{\delta} \rightarrow \mu\left(\right.$ tight $\left.C_{0}^{*}\right)$ iff $f_{\delta}^{A} \rightarrow \mu^{A}$ (tight $\left.C_{0}^{*}\right)$. $\mathbf{R}^{l_{1}+l_{2}}$.

(b) If $f_{i \delta} \rightarrow \mu_{i}$ (tight $C_{0}^{*}$ ) on $\mathrm{R}^{l_{i}}$ then $f_{1 \delta} \times f_{2} \delta \rightarrow \mu_{1} \times \mu_{2}$ (tight $C_{0}^{*}$ ) on

1.10 Theorem. Suppose $f_{\delta} \in L^{1}\left(\mathbf{R}^{n}\right)$ for $\delta>0$, and $f_{\delta}$ satisfies (c) and (d) of 1.7. Suppose further that $T$ is a subspace of $\mathbf{R}^{n}, \operatorname{dim}(T)=k$, and for some $f_{0}^{k} \in L^{1}(T)$,

(a) $\lim _{\delta \rightarrow 0} \int_{\{\operatorname{dist}(Y, T)>\epsilon\}}\left|f_{\delta}\right| d Y=0 \quad \forall \epsilon>0$;

(b) $\lim _{\delta \rightarrow 0} \int_{T^{1}} f_{\delta}\left(Y^{1}+Y^{2}\right) d \lambda^{n-k}\left(Y^{2}\right)=\int_{0}^{k}\left(Y^{1}\right)$, in the $L^{1}(T)$ topology.

Then $\lim _{\delta \rightarrow 0} f_{\delta}=\mu$ (tight $C_{0}^{*}$ ), where for any $g \in C_{0}\left(\mathbf{R}^{n}\right)$,

(c) $\int g d \mu=\int_{T} g\left(Y^{1}\right) f_{0}^{k}\left(Y^{1}\right) d \lambda^{k}\left(Y^{1}\right)$.

Proof. Here, and henceforth, $\lambda^{m}$ will denote $m$-dimensional induced Lebesgue measure.

(a) and 1.7(d) imply that we may restrict our attention to the integral over $C=C^{k} \oplus \bar{B}^{n-k}(0 ; \epsilon)$ for some large compact $C^{k} C T$ and $\bar{B}^{n-k}(0 ; \epsilon)$, the $\epsilon$-ball about 0 in $T^{\perp}$, for arbitrarily small positive $\epsilon$. In fact, by uniform continuity, we may choose $\epsilon$ so that $\left|g\left(Y^{1}+Y^{2}\right)-g\left(Y^{1}\right)\right| \leq \delta \quad \forall Y^{1} \in C^{k}, Y^{2} \in \bar{B}^{n-k}(0 ; \epsilon)$, given an arbitrary $\delta>0$. By (a) we may replace $T^{\perp}$ in the integral in (b) by $\bar{B}^{n-k}(0 ; \epsilon)$. The rest follows if we set, for $\pi_{T}$ the orthogonal projection onto $T$, $g(Y)=\chi_{C}(Y) g\left(\pi_{T} Y\right)+\chi_{C}(Y)\left[g(Y)-g\left(\pi_{T} Y\right)\right]+\left[1-\chi_{C}(Y)\right] g(Y)$.

C. The almost everywbere convergence.

1.11 Definition. The function $f \in L^{1}\left(R^{n}\right)$ is called radially almost everywhere fast-decreasing (RAEFD) if it has a majorant $k \in L^{1}$ of the form

$$
k=\sum_{j} c_{j} \chi_{R_{j}}
$$

where

(a) $c_{j} \geq 0 \quad \forall j$

(b) for all $j, R_{j}$ is a linear transform of the unit cube $\left\{|Y|_{\infty} \leq 1\right\}$;

(c) $3 K<\infty, a>0$ such that, for each integer $m \geq 0$,

$$
\sum_{j \in N_{m}} \lambda_{j} \leq K 2^{-a m}
$$

where $\lambda_{j}=c_{j} \lambda^{n}\left(R_{j}\right)=\left\|c_{j} \chi_{R_{j}}\right\|_{1}$, and $N_{m}=\left\{j: \lambda_{j} \leq 2^{-m}\right\}$.

A majorant of form (18) satisfying the conditions is called a rectangular majorant of $f$.

1.12 Definition. The family of functions $\left\{f_{\delta}: \delta>0\right\}$ is called specially majorized if each $f_{\delta}$ is RAEFD with rectangular majorant $k_{\epsilon}$ for some $\epsilon>0$, where 


$$
k_{\epsilon}=\left|\operatorname{det} A_{\epsilon}\right|\left(k_{1} \circ A_{\epsilon}\right) \quad \forall \epsilon>0,
$$

and the $A_{\epsilon}$ have the following properties:

(a) $A_{\epsilon}: \mathbf{R}^{n} \rightarrow \mathbf{R}^{n}$ is a nonsingular linear transformation for $\epsilon>0$;

(b) $A_{\delta} A_{\epsilon}=A_{\delta \epsilon}$;

(c) $\epsilon \geq 1 \Rightarrow A_{\epsilon} R_{j} \subset R_{j} \forall j$ in the sum (18) for $k_{1}$.

1.13 Claim. If the $f_{\delta}$ are specially majorized, then the maximal operator

is weak-type $(1,1)$.

$$
M: L^{1} \rightarrow \text { measurable functions, } \quad M b=\sup _{\delta>0}\left|b * f_{\delta}\right|,
$$

Proof. This follows at once from well-known results on maximal operators, i.e., Lemmas 2.1, 2.2, and 2.3 of [7].

1.14 Claim. Suppose $\lim _{\delta \rightarrow 0} f_{\delta}=\mu$ (tight $C_{0}^{*}$ ), and the maximal operator $M$ of (21) is weak-type $(1,1)$. Then for $b \in L^{1}\left(\mathbf{R}^{n}\right)$,

$$
\lim _{\delta \rightarrow 0}\left(b * f_{\delta}\right)(Y)=(b * \mu)(Y) \text { for a.e. } Y \in \mathbf{R}^{n}
$$

Proof (Cf. [3], [7].) Set $b=b_{n}+b_{n}^{\prime}, b_{n} \in C_{00},\left\|b_{n}^{\prime}\right\|_{1} \leq 4^{-n}$. For $b_{n}$, the convergence of (22) holds everywhere, and $\lambda^{n}\left\{Y: !\left|b_{n}^{\prime} * f_{\delta}\right|(Y)>2^{-n}\right.$ some $\left.\delta>0\right\}$ $\leq C 2^{-n}$. The result follows a.e. on letting $n \rightarrow \infty$.

1.15 Theorem. Let $C$ be a nonempty set. Suppose, for each $P \in C$, $\lim _{\delta \rightarrow 0} f_{\delta}^{P}=\mu^{P}$ (tight $C_{0}^{*}$ ), where the $f_{\delta}^{P}$ are specially majorized, independently of $P$, by rectangular majorants $k_{\epsilon(\delta)}$, such that, for the subspace $T \subset \mathbf{R}^{n}$, $\operatorname{dim}(T)=k$ :

(a) $A_{\epsilon}=I_{T} \oplus\left(A_{\epsilon}^{\prime}\right) T^{\perp}$;

(b) $R_{j}=R_{j}^{1} \oplus R_{j}^{2} \forall j, R_{j}^{1} \subset T, R_{j}^{2} \subset T^{\perp}$;

(c) $\exists \epsilon_{0}>0 \cdot \ni \cdot \lambda^{k}\left(R_{j}^{1}\right) \geq \epsilon_{0} \forall j$.

Then, if we define $\left(b * f_{\delta}\right)_{Y^{2}}\left(P, Y^{1}\right)=\left(b * f_{\delta}^{P}\right)\left(Y^{1}+Y^{2}\right), Y^{1} \in T, Y^{2} \in T^{\perp}$, and similarly for $(b * \mu)_{Y^{2}}$, it follows that if $b \in L^{1}$, then, for almost every $Y^{2}$ $\in T^{\perp},(b * \mu)_{Y^{2}}$ is well defined a.e. and $\left(b * f_{\delta}\right)_{Y^{2}} \rightarrow(b * \mu)_{Y^{2}}$ uniformly on $C \times K$, where $K$ is any compact subset of $T$.

Proof. The hypotheses imply that $\lambda_{j}^{\perp} \leq \epsilon_{0}^{-1} \lambda_{j}$, where $\lambda_{j}^{\perp}=c_{j} \lambda^{n-k}\left(R_{j}^{2}\right)$; also $\operatorname{det} A_{\epsilon}^{\prime}=\operatorname{det} A_{\epsilon}$. If we set $k_{1}^{\perp}\left(Y^{2}\right)=\Sigma_{c}{ }_{j} \chi_{R}{ }^{2}\left(Y^{2}\right)$ and $k_{\epsilon}^{\perp}=\left|\operatorname{det} A_{\epsilon}^{\prime}\right|\left(k_{1}^{\perp} \circ A_{\epsilon}^{\prime}\right)$, then $k_{\epsilon}^{\perp}$ satisfies the condition of 1.11 on $T^{\perp}$. Also, $k_{\epsilon}^{\perp}\left(Y^{2}\right) \geq$ $\sup \left\{k_{\epsilon}\left(Y^{1}+Y^{2}\right): Y^{1} \in T\right\}$. This implies $\sup \left\{\left|b * k_{\epsilon}\right|\left(Y^{1}+Y^{2}\right): \epsilon>0, Y^{1} \epsilon T\right\}$ satisfies a weak-type inequality. The theorem now follows from the $C_{\infty}$ case as in the proof of 1.14 . 
1.16 Claim. (a) Suppose $B: \mathbf{R}^{n} \rightarrow \mathbf{R}^{n}$ is nonsingular, and $f_{\delta}\left[f_{\delta}^{P}\right]$ satisfy the bypotheses of 1.12 [1.15]. Then the conclusions of the respective theorems bold for $f_{\delta}^{B}\left[\left(f_{\delta}^{P}\right)^{B}\right]$, defined as in 1.9. In 1.12, we may set $R_{j}^{B}=B R_{j}, A_{\epsilon}^{B}=$ $B A_{\epsilon} B^{-1}$, and the bypotheses also bold.

(b) With notation as in 1.9(b), the truth of the bypotbeses of 1.12 [1.15] carries over to direct products, for $k_{\epsilon}=k_{1 \epsilon} \times k_{2 \epsilon}, A_{\epsilon}=A_{1 \epsilon} \oplus A_{2 \epsilon}$, and $a<$ $\min \left(a_{1}, a_{2}\right)$.

(c) If $f_{j} \rightarrow \mu_{j}$ satisfy the bypotheses of $1.14,1 \leq j \leq m$, and $\lim _{\delta \rightarrow 0} f_{\delta}=$ $\mu\left(\right.$ tight $\left.C_{0}^{*}\right)$, with

$$
\left|f_{\delta}\right| \leq N \sum_{j}\left|f_{j}\right|, \quad N<\infty,
$$

then $f_{\delta} \rightarrow \mu$ also satisfy the bypotheses of 1.14 .

(d) If $f_{j \delta}^{P} \rightarrow \mu_{j}^{P}$ satisfy the bypotheses of 1.15, $j$ as above, $P \in C$, for the same subspace $T$ each $j$, and $\lim _{\delta \rightarrow 0} f_{\delta}^{P}=\mu^{P}$ with (23) bolding, then $f_{\delta}^{P}$ satisfy the conclusion of 1.15 for subspace $T$.

Proof. (c) follows by Lemma 2.3 [7]. The same method, applied to majorants of form $k_{\epsilon}^{\perp}$, yields (d). The others are straightforward.

2. The tight $C_{0}^{*}$ limit of the Poisson kernel at boundary points. Our approach will be to apply linear transformations that exchange limiting motion of a point for limiting change in a cone. We will not attempt the greatest generality, but will restrict ourselves to points at which the cone boundary, in "rounded" directions, has positive curvature.

If $A, B \subset \mathbf{R}^{n}$ and $P \in \mathbf{R}^{n}$, we say " $A \subset B$ locally at $P$ " if for some neighborhood $N$ of $P, A \cap N \subset B \cap N$. For any nonempty convex set $C$ its "inner part," Inn $(C)$. is defined to be the interior of $C$ as a subspace of the affine space generated by $C$. We note $\bar{C}=\overline{\operatorname{Inn}(C)}$.

If $\Gamma \subset \mathbf{R}^{n}, P \in \bar{\Gamma}$, let $T_{1}(\Gamma, P)$ be the largest subspace of $\mathbf{R}^{n}$ such that $T_{1} \subset \bar{\Gamma}$ locally at $P$. The (not necessarily proper) cone $\bar{\Lambda}(\Gamma, P)$ is defined to be the intersection of all closed half-spaces $\overline{\mathcal{H}}$ such that $\bar{\Gamma} \subset \overline{\mathcal{H}}$ and $P \in \partial \overline{\mathcal{H}}$. (If $P \in \Gamma$ we set $\bar{\Lambda}=\mathbf{R}^{n}$.) We define $T_{2}(\Gamma, P)$ to be the largest subspace such that $T_{2} \subset \bar{\Lambda}$ locally at $P$. It follows that $T_{2} \subset \bar{\Lambda}$ and $T_{1} \subset T_{2}$. We express the number of independent "flat," "rounded," and "sharp" directions of $\bar{\Gamma}$ at $P$ by $k(\Gamma, P)=\operatorname{dim}\left(T_{1}\right), r(\Gamma, P)=\operatorname{dim}\left(T_{2}\right)-\operatorname{dim}\left(T_{1}\right)$, and $k^{*}(\Gamma, P)=n-\operatorname{dim}\left(T_{2}\right)$, respectively.

It is clear that $\bar{\Lambda}=T_{2} \oplus \bar{\Lambda}_{P}(\Gamma)$, where $\Lambda_{P}(\Gamma)=\operatorname{Inn} \bar{\Lambda}_{P}(\Gamma)$ is a proper cone of $T_{2}^{\perp}$ Let us define $\Gamma_{P}=\operatorname{Inn} \bar{\Gamma}_{P}$ where $\bar{\Gamma}_{P}=T_{1}(\Gamma, P) \cap \bar{\Gamma}$. Then we get the following relations at once (see [2], [4]). (In this and the following, all duals of lower-dimensional cones are taken in the subspace which they generate.) 
2.1 Proposition. Suppose $\Gamma \subset \mathbf{R}^{n}, P \in \bar{\Gamma}$. Then $\bar{\Lambda}_{P}^{*} \subset \bar{\Gamma}^{*}$, and for $Q$ any point of $\Lambda_{P}^{*}$ the following bold:

(a) $k, r, k^{*}$ are nonnegative integers with sum $n$ at $P$.

(b) $T_{2}^{\perp}(\Gamma, \dot{P})=T_{1}\left(\Gamma^{*}, Q\right) ; k^{*}(\Gamma, P)=k\left(\Gamma^{*}, Q\right)$.

(c) If $A: \mathbf{R}^{n} \rightarrow \mathbf{R}^{n}$ is nonsingular, then $T_{1}(A \Gamma, A P)=A T_{1}(\Gamma, P)$, $T_{2}(A \Gamma, A P)=A T_{2}(\Gamma, P),(A \Gamma)_{A P}=A\left(\Gamma_{P}\right), \bar{\Lambda}(A \Gamma, A P)=A \bar{\Lambda}(\Gamma, P)$.

(d) If $\mathbf{R}^{n}=\mathbf{R}^{l_{1}} \oplus \mathbf{R}^{l_{2}}, \Gamma_{i} \subset \mathbf{R}^{l_{i}}, P_{i} \in \bar{\Gamma}_{i}$, then $\Gamma=\Gamma_{1} \oplus \Gamma_{2}$ is a proper cone of $\mathbf{R}^{n}, \Gamma^{*}=\Gamma_{1}^{*} \oplus \Gamma_{2}^{*}$, and at $P=P_{1}+P_{2}, T_{1}, T_{2}, \vec{\Lambda}, \Lambda_{P}$ and $\Gamma_{P}$ are all direct sums of the respective sets belonging to $P_{i}$ in $\mathbf{R}^{l_{i}}$.

(e) The parameters $k, r$, and $k^{*}$ are unchanged in (c) and summed in (d).

The following definition is used for our cone limiting operations:

2.2 Definition. The cone metric distance between two proper cones $\Gamma_{1}$ and $-\Gamma_{2}$ of $R^{n}$ is defined to be

$$
\operatorname{dist}\left(\Gamma_{1}, \Gamma_{2}\right)=d_{H}\left(\bar{\Gamma}_{1} \cap \bar{B}, \bar{\Gamma}_{2} \cap \bar{B}\right)
$$

where $\bar{B}$ is the closed unit ball and $d_{H}$ is the Hausdorff metric for closed sets:

$$
d_{H}(A, B)=\max \left\{\sup _{X \in A} \operatorname{dist}(X, B), \sup _{Y \in B} \operatorname{dist}(Y, A)\right\} .
$$

This is a metric, and it is clear that convergence in this metric implies local convergence in measure, or convergence in measure on compact sets. (The converse also holds for proper cones.) The following lemma is useful.

2.3 Lemma. (a) Let $A: \mathbf{R}^{n} \rightarrow \mathbf{R}^{n}$ be nonsingular, $\Gamma_{i} \subset \mathbf{R}^{n},\|\cdot\|$ the operator bound. Then

$$
\begin{aligned}
& \operatorname{dist}\left(A \Gamma_{1}, A \Gamma_{2}\right) \leq\|A\|\left\|A^{-1}\right\| \operatorname{dist}\left(\Gamma_{1}, \Gamma_{2}\right) ; \\
& \operatorname{dist}\left(A \Gamma_{1}, \Gamma_{1}\right) \leq \max \left\{\|I-A\|,\left\|I-A^{-1}\right\|\right\} .
\end{aligned}
$$

(b) Suppose $\Gamma_{i j} \subset \mathbf{R}^{l_{i}}, \Gamma_{j}=\Gamma_{1 j} \oplus \Gamma_{2 j}$. Then in $\mathbf{R}^{l_{1}} \oplus \mathbf{R}^{l_{2}}$,

$$
\operatorname{dist}\left(\Gamma_{1}, \Gamma_{2}\right) \leq \operatorname{dist}\left(\Gamma_{11}, \Gamma_{12}\right)+\operatorname{dist}\left(\Gamma_{21}, \Gamma_{22}\right) \text {. }
$$

(c) If $\Gamma_{j}$ are as in (a), then dist $\left(\Gamma_{1}^{*}, \Gamma_{2}^{*}\right)=\operatorname{dist}\left(\Gamma_{1}, \Gamma_{2}\right)$.

Proof. (a) and (b) follow directly from the definition of cone metric, if we use the fact that if $|P| \leq 1,|Q| \geq 1$, then $\left.|P-| Q\right|^{-1} Q|\leq| P-Q \mid$. Since $\Gamma^{* *}=$ $\Gamma$, to prove (c) we need only prove " $\leq$ "; and this follows using, for $P \notin \Gamma^{*}$, $\operatorname{dist}\left(P, \bar{\Gamma}^{*}\right)=-\min \{(P, X): X \in \bar{\Gamma} \cap \overline{\bar{B}}\}$.

2.4 Definition. If $T$ is a subspace of $\mathbf{R}^{n}, k=\operatorname{dim}(T)$, and $\Gamma \subset \mathbf{R}^{n}$, we define for $x \in \Gamma, Y^{1} \in T, P_{X}=P_{\Gamma ; X}$, 


$$
P_{\Gamma ; X}^{T}\left(Y^{1}\right)=\int_{T}^{\perp} P_{X}\left(Y^{1}+Y^{2}\right) d \lambda^{n-k}\left(Y^{2}\right)
$$

2.5 Theorem (kernel continuity). Let $\Gamma_{0},\left\{\Gamma_{\delta}\right\}_{\delta>0}$ be proper cones of $\mathbf{R}^{n}$ such that $\lim _{\delta \rightarrow 0} \Gamma_{\delta}=\Gamma_{0}$. Let $T$ be any linear subspace of $\mathbf{R}^{n}, k=\operatorname{dim}(T)$, $X, X_{0} \in \Gamma_{0}$. Then the following bold:

(a) $K \Gamma_{0 ; X}$ is continuous in the $L^{p}$ norms, $2 \leq p \leq \infty$, as a function of $X$. $\lim _{\delta \rightarrow 0} K_{\Gamma_{\delta ;} X_{0}}=K_{\Gamma_{0} ; X_{0}}$ in the same norms.

(b) $P \Gamma_{0}^{T} X$ is continuous in the $L^{p}$ norm, $1 \leq p \leq \infty$, as a function of $X$. $\lim _{\delta \rightarrow 0} P_{\Gamma \delta ; X_{0}}^{T}=P_{\Gamma_{0} ; X_{0}}^{T}$ in the same norms.

(c) The above statements bold with " $L$ " replaced by " $C_{0}$ "

Proof. (c) follows immediately from (a) and (b) since $K$ and $P$ are continuous and $L^{p}$ for some $p<\infty$. To prove (a) and (b) it is necessary to prove them only for the extreme norms.

(a) Case $p=2$. By Plancherel's theorem the claims will hold if they hold for $\hat{K}$. The first claim is obvious from (6), and the second follows from dominated convergence.

Case $p=\infty$. This follows from case $p=1$ for $\hat{K}$ as above.

(b) Case $p=1$. We need only consider the case $T=\{0\}$. The claims then follow from Case $p=2$ of $(a)$, since $f_{\delta} \rightarrow f_{0}\left(L^{2}\right) \Rightarrow f_{\delta}^{2} \rightarrow f_{0}^{2}\left(L^{1}\right)$.

Case $p=\infty$. We have

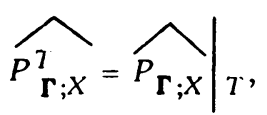

and the $L^{1}$ convergence of the latter follows easily using dominated convergence.

The limit cone we will use will be the "derivative" defined below, which does not necessarily have anything to do with ordinary concepts of differentiability.

2.6 Definition. Suppose $\Gamma \subset \mathbf{R}^{n}, P \in \bar{\Gamma}, \delta>0$. We define the standard differentiator $A_{\delta}=A_{\delta}(\Gamma, P)$ to be the linear transformation

$$
A_{\delta}=I_{T_{1}} \oplus \delta^{-1 / 2} I_{S_{2}} \oplus \delta^{-1} I_{S_{3}},
$$

where $T_{1}=T_{1}(\Gamma, P), S_{2}=T_{1}(\Gamma, P)^{\perp} \cap T_{2}(\Gamma, P), S_{3}=T_{2}(\Gamma, P)^{\perp}$. If there exists a proper cone $\Gamma_{0}$ such that

$$
\lim _{\delta \rightarrow 0} A_{\delta} \Gamma=\Gamma_{0}
$$

we call $P$ a "nice" point of $\Gamma$ and $\Gamma_{0}$ the derivative of $\Gamma$ at $P$.

2.7 Claim. Suppose $P$ is "nice," notation as in 2.6. Then

(a) $A_{\delta} \Gamma_{0}=\Gamma_{0} \forall \delta>0 ; A_{\delta} \Gamma_{0}^{*}=\Gamma_{0}^{*}$. 
(b) Suppose $B: \mathbf{R}^{n} \rightarrow \mathbf{R}^{n}$ is nonsingular and invariant on $T_{1}$ and $T_{2}$,

$$
B=\left(\begin{array}{lll}
B_{1} & C_{2} & D_{3} \\
0 & B_{2} & C_{3} \\
0 & 0 & B_{3}
\end{array}\right)
$$

in $\left(T_{1}, S_{2}, S_{3}\right)$-matrix block form. Then $B P$ is a "nice" point of $B \Gamma$, and

$$
\lim _{\delta \rightarrow 0} A_{\delta} B \Gamma=B_{0} \Gamma_{0}
$$

where

$$
B_{0}=\left(\begin{array}{lll}
B_{1} & 0 & 0 \\
0 & B_{2} & 0 \\
0 & 0 & B_{3}
\end{array}\right)=\lim _{\delta \rightarrow 0} A_{\delta} B A_{\delta}^{-1}
$$

(c) If $P_{i}$ is a "nice" point of $\Gamma_{i} \subset \mathbf{R}^{l_{i}}, \Gamma=\Gamma_{1} \oplus \Gamma_{2}$, then $P=P_{1}+P_{2}$ is a "nice" point of $\Gamma$ with $A_{\delta}=A_{1 \delta} \oplus A_{2 \delta}$ and $\Gamma_{0}=\Gamma_{10} \oplus \Gamma_{20}$.

Proof. Since $A_{\delta} A_{\epsilon}=A_{\delta \epsilon}$ and $A_{\delta}=A_{\delta}$, (a) follows from (2.6). (b) follows from (35) and 23(a), using $A_{\delta} B \Gamma=\left(A_{\delta} B A_{\delta}^{-1}\right)\left(A_{\delta} \Gamma\right)$. (c) follows from 2.3(b).

2.8 Corollary. Let $A: \mathbf{R}^{n} \rightarrow \mathbf{R}^{n}$ be any nonsingular linear transformation. Then $P$ is a "nice" point of $\Gamma$ iff $A P$ is a "nice" point of $A \Gamma$.

Proof. Clearly Definition 2.6 is invariant under unitary transformations. One may thus transform $A$ so that it satisfies the hypotheses of $2: 7(\mathrm{~b})$.

We shall apply the above machinery to Poisson kernels. We define a type of convergence to $P \in \bar{\Gamma}$ which is unrestricted in "flat" coordinates, Stein-admissible ([7]: within a narrower paraboloid-shaped region) in "rounded" ones, and restricted in "sharp" ones.

2.9 Definition. Suppose $P$ is a "nice" point of $\Gamma$. Then the family $\left\{X_{\delta}\right.$ : $\delta>0\}$ converges admissibly to $P$ in $\Gamma$ if

(a) $\left\{X_{\delta}\right\} \subset \Gamma, \lim _{\delta \rightarrow 0} X_{\delta}=P$;

(b) there is a function $\epsilon:\{\delta>0\} \rightarrow\{\epsilon>0\}$ such that for some $\delta_{0}>0$ the closure of the set $\left\{A_{\epsilon(\delta)} X_{\delta}: 0<\delta \leq \delta_{0}\right\}$ is a compact subset of $\Gamma_{0}$.

The "nice" condition is a local requirement that, in the limit, the cone boundary be in each direction either flat, sharp, or "round" (parabola-shaped). However, this still allows certain "pathological" possibilities, which are eliminated by a condition on the dual:

2.10 Definition. The point $P \in \bar{\Gamma}$ is said to be regular if

(a) $P$ is a "nice" point of $\Gamma$; 
(b) $\bar{\Gamma}_{0}^{*} \cap\left(T_{1}(\Gamma, P)+Y\right)$ is either empty or a translate of a cone for every. $Y \in \mathbf{R}^{n}$.

We now state the central theorem of this section.

2.11 Theorem (Tight $C_{0}^{*}$ limit of the Poisson kernel). Suppose $\Gamma \subset \mathbf{R}^{n}, P \in$ $\bar{\Gamma}$ regular, and $\lim _{\delta \rightarrow 0} X_{\delta}=P$ admissible in $\Gamma$. Then

$$
\lim _{\delta \rightarrow 0} P_{\mathbf{r} ; \dot{X}_{\delta}}=\mu\left(\text { tight } C_{0}^{*}\right)
$$

where $\mu$ is defined by the following for any $g \in C_{0}\left(\mathbf{R}^{n}\right)$. Here $P_{\Gamma_{P ; P}}$ is the $k$ dimensional Poisson kernel $(=1$ if $k=0)$, defined on $T_{1} \approx \mathbf{R}^{k}$ :

$$
\int g d \mu=\int_{T_{1}(\mathbf{\Gamma}, P)} g\left(Y^{1}\right) P_{\Gamma_{P} ; P}\left(Y^{1}\right) d \lambda^{k}\left(Y^{1}\right) .
$$

Proof. We will first prove the following lemma:

Lemma A. Suppose $P$ is regular, other notation as above. Then

(a) $\bar{\Gamma}_{0}^{*}=\bar{\Gamma}_{P}^{*}+\phi(\Delta)$

where $\Delta=\pi_{T_{1}^{\perp}} \vec{\Gamma}_{0}^{*}$, where $\pi$ is the orthogonal projection, and $\pi_{T_{1}^{+}} \phi=\pi_{T_{1}^{\perp}}$ on $\Delta$.

Proof of Lemma A. We claim $\bar{\Gamma}_{P}^{*}=\bar{\Gamma}_{0}^{*} \cap T_{1}$. Since $\bar{\Gamma}_{P} \subset \bar{\Gamma}_{0}$, clearly $\bar{\Gamma}_{0}^{*} \cap$ $T_{1} \subset \bar{\Gamma}_{P^{\prime}}^{*}$. To show the opposite inclusion we need only show $\pi_{T_{1}} \bar{\Gamma}_{0} \subset \bar{\Gamma}_{P}$. Suppose $Q \in \bar{\Gamma}_{0}$. Then $A_{\eta} Q \in A_{\eta} \bar{\Gamma}_{0}=\bar{\Gamma}_{0} \forall \eta>0$, so $\lim _{\eta \rightarrow 0} A_{\eta} Q=\pi_{T_{1}} Q \in \bar{\Gamma}_{0}$. Similarly, one uses the $A_{\delta}, \delta \rightarrow 0$, to show that any $Q^{1} \in T_{1}-\bar{\Gamma}_{P}$ is bounded away from $A_{\delta} \bar{\Gamma}$ for small $\delta$, and hence $\notin \bar{\Gamma}_{0}$.

We have by assumption that, for each $Y^{2} \in \Delta, \quad \exists \mid \phi\left(Y^{2}\right) \cdot \ni \cdot\left(Y^{2}+T_{1}\right) \cap \bar{\Gamma}_{0}^{*}$ $=\phi\left(Y^{2}\right)+\bar{\Gamma}_{0}^{*}\left(Y^{2}\right)$, for $\bar{\Gamma}_{0}^{*}\left(Y^{2}\right)$ some cone of $T_{1}$. Since $\phi\left(Y^{2}\right) \in \bar{\Gamma}_{0}^{*}$ and $\bar{\Gamma}_{0}^{*} \subset \bar{\Gamma}_{0}^{*}$ we have $\bar{\Gamma}_{P}^{*} \subset \bar{\Gamma}_{0}^{*}\left(Y^{2}\right)$. However, since $\bar{\Gamma}_{0}^{*}$ and $\bar{\Gamma}_{0}^{*}\left(Y^{2}\right)$ are cones, we must have $\delta \phi\left(Y^{2}\right)+\vec{\Gamma}_{0}^{*}\left(Y^{2}\right) \subset \bar{\Gamma}_{0}^{*} \forall . S>0$, and hence $\bar{\Gamma}_{0}^{*}\left(Y^{2}\right) \subset \bar{\Gamma}_{P}^{*}$. This proves Lemma $A$.

Now, applying (7) and (30), we get, for $U \in T_{1}, X \in \mathbf{R}^{n}, X^{1}=\pi_{T_{1}}(X)$ :

$$
\begin{aligned}
& \widehat{P_{\mathbf{r}_{0} ; X}^{T_{1}}}(U)=\left\|K_{\mathbf{r}_{0} ; X}\right\|_{2}^{-2} \int_{H\left(\mathbf{r}_{0}^{*}, U\right)} e^{-4 \pi(X, Y)} d Y \\
& =\left\|K_{\mathbf{r}_{0 ;} ;}\right\|_{2}^{-2} \int_{\phi(\Delta)+H\left(\Gamma_{p}^{*}, U\right)} \exp \left[-4 \pi\left(X, \pi_{T_{1}} Y\right)\right] \exp \left[-4 \pi\left(X, \pi_{\left.\left.T_{1}^{\perp}\right)\right]}\right.\right. \\
& =\left\{\left\|K_{\Gamma_{0} ; X}\right\|_{2}^{-2} \int_{\Delta} e^{-4 \pi\left(X, \phi\left(Y^{2}\right)\right)} d \lambda^{n-k}\left(Y^{2}\right)\right\} \int_{H}\left(\Gamma_{p}^{*}, U\right) e^{-4 \pi\left(X^{1}, Y^{1}\right)} d \lambda^{k}\left(Y^{1}\right) \\
& =\overbrace{P P_{P ; X^{1}}}(U) \text {. }
\end{aligned}
$$


But $c$ must equal 1 , since both functions are continuous and take the value 1 at 0 ; and it follows by continuity that

$$
P_{\Gamma_{0 ; X}}^{T_{1}}=P_{\Gamma_{P} ; \pi_{T_{1}} X} \text { everywhere on } T_{1} .
$$

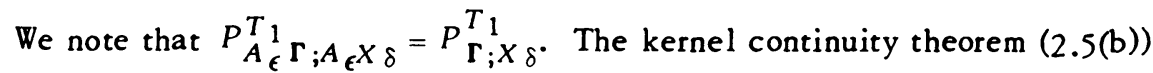
now implies that the hypotheses of Theorem 1.10 are satisfied for $f_{\delta}=P_{r_{i} X \delta}$, $T=T_{1}$, and our theorem follows.

\subsection{Corollary ( $L^{1}$ limit of Poisson integral at boundary). Suppose the} bypotheses of 2.11 are satisfied, and $b \in L^{1}\left(\mathbf{R}^{n}\right)$. For $Y^{2} \in T_{1}$ define $b_{Y^{2}}$ on $T_{1} \approx \mathbf{R}^{k}$ by $b_{Y^{2}}\left(Y^{1}\right)=b\left(Y^{1}+Y^{2}\right)$. Then $b_{Y^{2}}$ is defined and $L^{1}$ for a.e. $Y^{2}$, and if we define

$$
\left(b * P_{\mathbf{r}_{;} P}\right)\left(Y^{1}+Y^{2}\right)=\left(b_{Y^{2}} * P_{\mathbf{r}_{P ; P}}\right)\left(Y^{1}\right),
$$

the right-band convolution being of $\mathbf{R}^{k}$, we get

$$
\lim _{\delta \rightarrow 0} b * P_{\Gamma ; X}=b * P_{\Gamma ; P}, \text { in the } L^{1}\left(\mathbf{R}^{n}\right) \text { norm. }
$$

Proof. Theorem 2.11 and Lemma 1.8 .

To apply 2.11 and 2.12 , we have to show that significant classes of cones have regular points. The results below are to this end.

2.13 Claim. (a) Suppose $A: \mathbf{R}^{n} \rightarrow \mathbf{R}^{n}$ is nonsingular and $P \in \bar{\Gamma}$ is regular. Then $A P$ is a regular point of $A \Gamma$. $\bar{\Gamma}_{1} \oplus \bar{\Gamma}_{2}$.

(b) Suppose $P_{i} \in \bar{\Gamma}_{i} \subset \mathbf{R}^{l_{i}}$ are regular. Then $P_{1}+P_{2}$ is a regular point of

Proof. To prove (a) we transform by unitary transformations as in 2.8 and note that, by the invariance of $B_{0}$ on $T_{1}, S_{2}$, and $S_{3},\left(B_{0} \Gamma_{0}\right) *=B_{0}^{\#} \Gamma_{0}^{*}$ satisfies $2.10(\mathrm{~b})$ if $\Gamma_{0}^{*}$ does. (b) is elementary using $2.1(\mathrm{~d})$.

2.14 Lemma. If $P \in \bar{\Gamma}$ and $r(\Gamma, P)=0$ then $P$ is regular.

Proof. We may assume $k<n$, since the result is obvious for $P \in \Gamma$. The assumption implies $T_{1}=T_{2}$, and $A_{\epsilon}=I_{T_{1}} \oplus \epsilon^{-1} I_{T_{1}}$. It is clear that $A_{\epsilon} \bar{\Gamma} \cap T_{1}$ $=\bar{\Gamma} \cap T_{1}=\bar{\Gamma}_{P} \forall \epsilon$. We claim that, for any $\Gamma$ and $P \in \partial \bar{\Gamma}, \bar{\Lambda}_{P}(\Gamma)=\pi_{T} \perp \bar{\Gamma}$. In fact, it is clear that $\pi_{T} \frac{\perp}{\Gamma}$ is a cone and contained in $\bar{\Lambda}_{P}(\Gamma)$; but also, since $\bar{\Gamma} \subset T_{2} \oplus_{T^{\frac{1}{2}}} \bar{\Gamma}$, we have $\bar{\Lambda}_{P}(\Gamma) \subset \pi_{T} \frac{1}{2} \bar{\Gamma}$. In our case, the consequence of this fact is that $\bar{\Lambda}_{P}(A \Gamma)=\bar{\Lambda}_{P}(\Gamma)$, and hence we get, using an argument like that of the proof of Lemma $A$ to 2.11 , a long with a compactness argument, that 

$\underset{\epsilon \rightarrow 0}{\lim \sup _{\epsilon}} A_{\epsilon} \bar{\Gamma} \subset \bar{\Gamma}_{P} \oplus \bar{\Lambda}_{P}(\Gamma), \quad$ in the cone metric sense.

On the other hand, since $\bar{\Gamma}_{P} \subset \bar{\Gamma}$ and $\pi_{T} \frac{1}{1} \bar{\Gamma}=\bar{\Lambda}_{P}$, we have, for any $Q^{1} \epsilon$ $\bar{\Gamma}_{p}, Q^{2} \in \bar{\Lambda}_{P}$, there exists $Q^{\prime} \in T_{1}$ such that $Q^{1} \in \bar{\Gamma}$ and $Q^{\prime}+Q^{2} \in \bar{\Gamma}$, hence by convexity, for every $\epsilon>0, Q^{1}+\epsilon Q^{\prime}+\epsilon Q^{2} \epsilon \bar{\Gamma}$ and $Q^{1}+\epsilon Q^{\prime}+Q^{2} \epsilon A \bar{\Gamma}$. Hence, taking the limit, we get

$$
\bar{\Gamma}_{P} \oplus \bar{\Lambda}_{P}(\Gamma) \subset \underset{\epsilon \rightarrow 0}{\lim \inf } A_{\epsilon} \bar{\Gamma}, \quad \text { in the cone metric sense. }
$$

Hence $P$ is "nice," and regularity follows easily by $2.1(\mathrm{~d})$.

2.15 Lemma. If $P$ is "nice" and $k(\Gamma, P)=1$ then $P$ is regular.

Proof. 2.10(b) follows because $\left(T_{1}+Y\right) \cap \bar{\Gamma}_{0}^{*}$ must be empty or a half-line for any $Y$.

2.16 Definition. $\Gamma$ is called polytopic [simplicial] if a section of $\bar{\Gamma}$ is a polytope [simplex]. It is called $C^{n}$ if $\partial \Gamma$ is a $C^{n}$ hypersurface at every nonzero point, and regular if every point of $\bar{\Gamma}$ is regular.

2.17 Corollary. If $P=0$ or $P \in \Gamma, P$ is regular. If $\Gamma$ is polytopic it is regular. If $\Gamma$ is $C^{2}$ and satisfies the sharp curvature condition (Definition 3.1), it is regular.

Proof. The first two claims follow from 2.14, the last from 2.15.

Comment. The case of points which are "nice" but not regular may be of interest. Such points may be constructed, for instance, by constructing a cone $\Gamma^{*}$ which satisfies $2.7(\mathrm{a})$ but not $2.10(\mathrm{~b})$, and then taking its dual. It may easily be concluded that a radial limit of the form of 1.10 exists, but it apparently does not equal $P_{\Gamma_{P} ; P}$, and may even differ along different radii. It is possible that many positive reproducing kernels of type (4) for $H^{P}\left(\Omega_{\Gamma_{P}}\right)$ may be constructed in this way, for $\operatorname{dim}\left(\Gamma_{P}\right)>1$.

3. Decrease at infinity of Poisson kernels. The almost everywhere convergence of the Poisson integral.

A. Shape of certain Poisson kernels. Any simplicial cone is a linear transform of the octant, which is a direct sum of copies of $(0, \infty)$, so its Poisson kernel is the direct product of $P_{(0, \infty) ; x_{i}}\left(y_{i}\right)=x_{i} / \pi\left(x_{i}^{2}+y_{i}^{2}\right)$. Since the dual of a polytopic cone is a polytopic cone, every polytopic cone is the special intersection of simplicial cones. A condition on the decrease of $P$ at $\infty$ follows by 1.6; in fact, using (3), (9), (16), and $\left\|K_{\Gamma ; X}\right\|_{2}^{2}=K_{\Gamma ; 2 X}(0)$, an.expliciţ formula can be written.

The other case we consider is that of a smooth, "rounded" cone. 
3.1 Definition. $\Gamma \subset \mathbf{R}^{n}$ satisfies the flat curvature condition if, for some proper circular cone $\Delta$ of $\mathbf{R}^{n}$ and every $P \in \partial \Gamma$, there is a rotation $\rho_{P}$ of $\mathbf{R}^{n}$ such that $P \in \partial\left(\rho_{P} \Delta\right)$ and $\rho_{P} \Delta \subset \Gamma$. The sharp curvature condition is stated similarly but reverses the last inclusion.

3.2 Claim. The two curvature conditions of 3.1 are dual.

Proof. The flat condition is equivalent to the condition that the proper cone $\Gamma$ be the convex hull of a set of form $\left\{\rho_{a} \Delta: a \in \mathrm{A}\right\}, \Delta$ a fixed circular cone, and the $\rho_{a}$ rotations. The sharp condition is equivalent to the requirement that $\Gamma$ be the intersection of such a set. (The proofs are exercises in elementary convex set theory; we leave them to the reader.) But these two conditions are dual to each other, and 3.2 follows.

3.3 Theorem (Decrease of Poisson kernels of "rounded" cones). Suppose $\Gamma \subset \mathbf{R}^{n}$ and

(a) $n=3$ and $\Gamma$ satisfies the flat curvature condition, or

(b) $n \geq 4, \Gamma$ is $C^{[n / 2]}$, and $\Gamma$ satisfies the sharp curvature condition. Then, if we define $m_{\mathbf{r}}$ on $\mathbf{R}^{n}$ by

$$
m_{\mathbf{r}} \boldsymbol{r}^{\prime}(Y)=\left\{\begin{array}{l}
1 \quad \text { if }|Y| \leq 1 \\
|Y|^{-n / 2}[\max \{1, \operatorname{dist}(Y, \partial \Gamma U-\partial \Gamma)\}]^{-1 / 2} \text { if }|Y| \geq 1,
\end{array}\right.
$$

there exists for every compact set $D \subset \Gamma$ a constant $N<\infty$ such that

$$
\left|K_{\mathbf{r} ; X}\right| \leq N m_{\mathbf{r}} \quad \forall X \in D,
$$

and bence $N_{2}<\infty$ such that

$$
P_{\mathbf{\Gamma} ; X} \leq N_{2} m_{\Gamma}^{2} \quad \forall X \in D .
$$

Proof. The claim for the Poisson kernel follows from the one for the Szegö kernel. To prove the latter, we integrate (1) radially to get

$$
K_{\Gamma_{;} X}(Y)=(2 \pi)^{-n}|X|^{-1}(n-1) ! \int_{C_{X}^{*}}(1+i(Y, \sigma))^{-n} d \lambda^{n-1}(\sigma) .
$$

Setting $M(X)=(2 \pi)^{-n}|X|^{-1}(n-1)$ ! we get for $p \neq 0$

$$
K_{\mathbf{r} ; X}(p Y)=M(X) p^{-n} \int_{C_{X}^{*}}(1 / p+i(Y, \sigma))^{-n} d \lambda^{n-1}(\sigma) .
$$

Since $M(X)$ is continuous in $X$ we can neglect it in our considerations; and since the estimate obviously holds for $|Y| \leq 1$ in (46), our task is reduced to showing that, for all $X \in D,|Y|=1$, and $p \geq 1$, and some $N_{3}$ 


$$
\begin{aligned}
\left|\int_{C_{X}^{*}}(1 / p+i(Y, \sigma))^{-n} d \lambda^{n-1}(\sigma)\right| & \leq N_{3} p^{n} m_{\Gamma}(p Y) \\
& =N_{3} p^{n / 2}[\max \{1, p \operatorname{dist}(Y, \partial \Gamma U-\partial \Gamma)\}]^{-1 / 2}
\end{aligned}
$$

From the hypotheses it follows (since a $C^{2}$ cone always must satisfy the flat curvature condition) that $\Gamma^{*}$ satisfies the sharp condition, or, equivalently, there exists a ball $\bar{B}_{4}$ of $\mathbf{R}^{n-1}$, with radius $N_{4}$, such that for each $X \in D$ and $P \in \partial C_{X}^{*}$, there is a translation $\tau_{X, P}=\tau$ such that $P \in \partial\left(\tau \bar{B}_{4}\right)$ and $C_{X}^{*} \subset \tau \bar{B}_{4}$. Here we identify $\{V:(X, V)=1\}$ with $R^{n-1}$ by an affine isometry。Also, for $n \geq 4$, the sharp condition on $\Gamma$ implies that $\Gamma^{*}$ will also be $C^{[n / 2]}$, and hence so will $\partial C_{X}^{*}$, continuously in $X$.

We simplify (47) by Fubini's theorem. Define $[a, b]=[a(X, Y), b(X, Y)]=$ $\left(Y, C_{X}^{*}\right)$ and $\theta(y)=\theta(X, Y, y)=\lambda^{n-2}\left(\left\{\sigma \in C_{X}^{*}:(Y, \sigma)=y\right\}\right)$; then we get

$$
\int_{C_{X}^{*}}(1 / p+i(Y, \sigma))^{-n} d \lambda^{n-1}(\sigma)=\int_{a}^{b}(1 / p+i y)^{-n} \theta(y) d y .
$$

Choosing $\epsilon_{0}=1 / 4 \min \left\{b-a: X \in D, Y \in S^{n-1}\right\}$, and noting that dist $(Y, \partial \Gamma U-\partial \Gamma)$ is comparable to $\min \{|a|,|b|\}$, we divide (48) into four cases:

(a) $a$ and $b$ have same sign, $\min \{|a|,|b|\} \geq \epsilon_{0}$;

(b) $a$ and $b$ have same sign (or one is 0 ), and $\min \{|a|,|b|\} \leq \epsilon_{0}$;

(c) $a$ and $b$ have opposite signs, $\min \{|a|,|b|\} \geq \epsilon_{0}$;

(d) $a$ and $b$ have opposite signs, $\min \{|a|,|b|\} \leq \epsilon_{0}$.

We note that in (a) and (c) we can equivalently replace the right of (48) by $N_{3}^{\prime} p^{n / 2-1 / 2}$. Then case (a) is obvious, since the integrand in (47) is bounded independently of $p$, and $\lambda^{n-1}\left(C_{X}^{*}\right)$ independently of $X$. For the other cases, we need the following lemma, whose proof is elementary.

3.4 Lemma. Suppose $m \geq 0$ and $a>0$. Then $3 C_{a, m}<\infty$ such that, for every $A, p>0$,

$$
\int_{0}^{A}(\max \{1, p x\})^{-m_{x}}{ }^{a-1} d x \leq p^{-a} Q_{\alpha, m}(A p)
$$

where

$$
Q_{a, m}(t)= \begin{cases}C_{a, m}(\min \{1, t\})^{a} & \text { if } a<m ; \\ C_{m, m}(1+\ln +t)(\min \{1, t\})^{m} & \text { for } a=m ; \\ C_{a, m} t^{a-m}(\min \{1, t\})^{m} & \text { if } a>m .\end{cases}
$$

To use the lemma, we note that the sharp curvature condition implies there exists $N_{5}<\infty$ such that, for $X \in D, Y \in S^{n-1}, a \leq y \leq b$,

$$
\theta(y) \leq N_{5}(\min \{y-a, b-y\})^{n / 2-1}
$$


(To get the in equality, we replace $\Gamma^{*}$ with the larger cone $\rho_{V_{1}} \Delta \cap \rho_{V_{2}} \Delta$ (cf. 3.1), where $V_{1}$ and $V_{2}$ are defined in the proof of (d) below.) Also we have, for $z \in \mathbf{C}$,

$$
\left|(1 / p+i z)^{-n}\right| \leq p^{n}(\max \{1, p|z|\})^{-n}
$$

(b) now follows, applying 3.4 for the case $m=n, a=n / 2$. The results we get for (a) and (b) are, in fact, the stronger ones of Corollary 3.5.

We now use $C^{[n / 2]}$ to prove (c) and (d) for $n \geq 4$. Because of the smoothness, we have $\phi(y)$ is $C^{[n / 2]}$ on $(a, b)$. Let $\widetilde{\theta}(y)$ be the $n-1$ degree polynomial approximation to $\theta(y)$ at $y=0$, and let $\theta_{r}(y)=\theta(y)-\tilde{\theta}(y)$ for $a \leq y \leq b$.

Case (c). There exists a constant $N_{6}<\infty$, such that, for all $X, Y$ satisfying (c),

$$
\begin{gathered}
\max \left\{|\tilde{\theta}(z)|: \operatorname{Re}(z) \in[a, b], \operatorname{Im}(z) \in\left[-\epsilon_{0}, 0\right]\right\} \leq N_{\sigma}, \\
\left|\theta_{r}(y)\right| \leq N_{G}|y|^{[n / 2]} \text { on }[a, b] .
\end{gathered}
$$

Letting $\chi$ be the contour going straight from $a$ to $-\epsilon_{0}$, from $-\epsilon_{0}$ to $\epsilon_{0}$ via the semicircle of radius $\epsilon_{0}$ under the $x$-axis, and straight from $\epsilon_{0}$ to $b$, we get $\left|\int_{a}^{b}(1 / p+i y)^{-n} \theta(y) d y\right| \leq\left|\int_{\gamma}(1 / p+i z)^{-n} \tilde{\theta}(z) d z\right|+N_{6} p^{n} \int_{a}^{b}(\max \{1, p|y|\})^{-n}|y|^{[n / 2]} d y$

$$
\leq 2 G N_{6} \epsilon_{0}^{-n}+2 N_{6} C[n / 2]+1, n p^{n-[n / 2]-1} \leq C^{\prime} p^{n / 2-1 / 2}
$$

by 3.4 , where $G=\max \left\{\operatorname{diam} C_{X}^{*}: X \in D\right\}$. This proves (c).

Case (d), $n \geq 4$. We define $V_{1}(X, Y)=V_{1}, V_{2}(X, Y)=V_{2}$ to be the unique points of $C_{X}^{*}$ such that $(Y, V)=a$ and $b$, respectively. Since not both $|a|$ and $|b|$ can be $\leq \epsilon_{0}$, we will assume-it is $|a|$. (The other case is similar.) Define the affine map $E^{*}=E_{X, Y}^{*}$ on $\mathbf{R}^{n-1} \approx\{V:(X, V)=1\}$ by setting

$$
E^{*}\left(V_{1}+\widetilde{V}+V^{\perp}\right)=V_{1}+|a|^{-1 / 2} \widetilde{V}+|a|^{-1} V^{\perp}
$$

where $\widetilde{V}$ is in the linear subspace of $\mathbf{R}^{n-1}$ parallel to the image of $\{(Y, V)=a\}$ $\cap\{(X, V)=1\}$, and $V^{\perp}$ is normal to this subspace.

The family $E^{*}\left(C_{X}^{*}\right)$ is clearly $C^{[n / 2]}$-continuous in $X \times Y$ for $X, Y$ satisfying (d), $|a| \leq \epsilon_{0}$. In fact, due to the differentiability and curvature of $C_{X}^{*}$, the map from $X \times Y$ to $E_{X, Y}^{*}\left(C_{X}^{*}\right)$ extends to the closure of $G=\{X \times Y: X, Y$ satisfy (d) $\left.{ }_{a}\right\}$, that is, to $\{|a|=0\}$, continuously in the sense of $C^{[n / 2]}$ local parametrizations of the boundary. The limiting set is the solid paraboloid whose boundary is the second-order approximation to $\partial C_{X}^{*}$ at $V_{1}$.

We can therefore apply compactness arguments to this family. We define $\theta_{2}(y)=\theta_{2}(X, Y, y)=\lambda^{n-2}\left(E^{*}\left\{V \in C_{X}^{*}:(Y, V)=|a| y\right\}\right) . \theta_{2}$ is defined on 
$\left[-1,|a|^{-1} b\right]$, and is $C^{[n / 2]}$ on the interior, continuously in $X \times Y$ on the closure of $G$. Also, since all the $E^{*}\left(C_{X}^{*}\right)$ are contained in a solid paraboloid whose boundary is tangent to $\partial C_{X}^{*}$ at $V_{1}$, it still satisfies, uniformly in $X \times Y$,

$$
\left|\theta_{2}(y)\right| \leq N_{5}(y+1)^{n / 2-1} \text {. }
$$

A change of variables in (49) yields

$$
\int_{a}^{b}(1 / p+i y)^{-n} \theta(y) d y=|a|^{-n / 2} \int_{-1}^{|a|^{-1} b}\left(1 /|a| p+i y^{\prime}\right)^{-n} \theta_{2}\left(y^{\prime}\right) d y^{\prime} .
$$

If we separate the integrals $\int_{-1}^{1}$ and $\int_{1}^{|a|-1 b}$, proceed as in (56) with the former, and estimate the latter crudely using (58), we get

$$
\left|\int_{a}^{b}(1 / p+i y)^{-n} \theta(y) d y\right| \mid \begin{aligned}
& \leq N_{7}|a|^{n / 2} p^{n} \leq N_{7} p^{n / 2} \text { if }|a| p \leq 1 ; \\
& \leq N_{7}|a|^{n / 2-[n / 2]-1} p^{n-[n / 2]-1} \leq N_{7}|a|^{-1 / 2} p^{n / 2-1 / 2} \\
& \text { if }|a| p \geq 1 .
\end{aligned}
$$

This is equivalent to our claim,

The cases (c) and (d) for $n=3$ are implied by the sharp curvature condition on $\Gamma^{*}$. We suppose (the other case is similar) that $|a| \leq|b|$. If we let $\widetilde{\theta}(y)=$ $(b-y) \theta(0) / b$, then $\left|\theta_{r}(y)\right| \leq 2 \theta(0)|y| /|a|$. (c) and (d) follow from (52). This completes the proof of 3.3 .

We note here that the $C^{[n / 2]}$ requirement could be relaxed to "uniformly Lipschitz $[n / 2]-1$ order derivatives"; this explains the apparent anomaly of the case $n=3$. Stronger differentiability conditions yield a better decrease, up to $C^{n}$ (or uniformly Lipschitz $n-1$ order derivatives), which yields the result below. This is the best possible, being the decrease found for a circular cone (see [6]):

3.5 Corollary. Suppose $n \geq 3$ and $\Gamma \subset \mathbf{R}^{n}$ is $C^{n}$ and satisfies the sharp curvature condition. Then the conclusions of 3.3 bold with $m_{\Gamma}$ replaced by

$$
\tilde{m}_{\mathbf{\Gamma}}(Y)=\left\{\begin{array}{l}
1 \quad \text { if }|Y| \leq 1 \\
|Y|^{-n / 2}(\max \{1, \operatorname{dist}(Y, \partial \Gamma U-\partial \Gamma)\})^{-n / 2} \text { if }|Y| \geq 1 .
\end{array}\right.
$$

Proof. Same as for 3.3, substituting the better smoothness conditions.

In summary, we note that the rate of decrease of $P_{\Gamma_{;} X}(p Y)$ as $p \rightarrow \infty$ for $Y$ fixed depends on whether $Y \in T_{1}(\Gamma, P)$ for some $P \in \partial \Gamma$ in both the polytopic and smooth cases. We express the following conjecture, which works in both these cases:

3.6 Conjecture. Suppose $\Gamma \subset \mathbf{R}^{n}$ is regular, and its boundary is a union of sufficiently smooth segments. Then, for $X \in \Gamma, Y \in S^{n-1}$, and $p$ large, we have 


$$
P_{\mathbf{r}_{;} X}(p Y)=O\left(p^{-s}\right)
$$

where

$$
s=\min \left\{2 k(\Gamma, p)+r(\Gamma, P): Y \in T_{1}(\Gamma, P)\right\}
$$

B. The almost everywhere convergence of Poisson integrals. We obtain results of this type only at points $P$ where $r(\Gamma, P)=0$. Any roundedness, even in the simplest cases (i.e. circular cone), results in complications which make impossible a proof of the existence of a weak $(1,1)$ maximal operator by the ordinary methods (such as $1.11,1.12$ ). The case $r(\Gamma, P)=0$ results in the convenience of 2.14, however, and the author conjectures that the conclusions of the following theorem hold for all such points, not only the special cases of the hypothesis.

3.7 Theorem. Suppose that $\Gamma \subset \mathbf{R}^{n}$ is derived by a finite number of linear transformations and direct summations from cones of the following form:

(a) any proper cone of $\mathbf{R}$ or $\mathbf{R}^{2}$, or

(b) any cone satisfying the bypotheses of Theorem 3.3.

Then if $P \in \bar{\Gamma}, r(\Gamma, P)=0, b \in L^{1}\left(\mathbf{R}^{n}\right)$, and $b_{Y^{2}}$ is as in 2.12 for $Y^{2} \in T_{1}(\Gamma, P)^{\perp}$, the following bolds for a.e. $Y^{2} \in T_{1}^{\perp}$ :

If $Z^{2}(\delta)=X^{2}(\delta)+i Y^{2}(\delta)$ converges restrictedly to $i Y^{2}$ as $\delta \rightarrow 0$ in the tube domain $\Omega_{P}^{\prime}=\Lambda_{P} \oplus i T_{1}^{\perp}$ of $T_{1}^{\perp} \oplus i T_{1}^{\perp} \approx \mathrm{C}^{n-k}$, and if we define

$$
P b_{Z^{2}(\delta)}\left(X^{1}, Y^{1}\right)=\left(b * P_{\mathbf{r}_{;} X^{1}+X^{2}(\delta)}\right)\left(Y^{1}+Y^{2}(\delta)\right)
$$

for $X^{1}, Y^{2} \in T_{1}$ and $X^{1}+X^{2}(\delta) \in \Gamma$, then

$$
\lim _{\delta \rightarrow 0} P b_{Z^{2}(\delta)}=\left(P b_{Y^{2}}\right) \Gamma_{P}
$$

uniformly on compact subsets of $\Gamma_{P} \times T_{1}$, where $P b_{Y^{2}}$ is the lower-dimensional Poisson integral, defined as in (4).

Proof. By 2.14 the a.e. limit, if it exists, must be the one given. Because of 1.16 and the continuity of the limiting functions of (65), which allows us to make the slight translations required to use 1.16(a) uniformly in $P$, we only have to show that we can apply 1.15 to $\Gamma=(0, \infty)$ and $\Gamma$ satisfying (b). Here the only cases to be proved are $P \in \Gamma(k=n)$ and $P=0(k=0)$. The differentiator $A_{\epsilon}$ is $I$ and $\epsilon^{-1} I$, respectively, and (1) implies $P_{\Gamma}(\delta X, \delta Y)=\delta^{-n} P_{\Gamma}(X, Y)$. To prove the functions of (64) satisfy the hypotheses of 1.15 , for $T=\mathbf{R}^{n}$ and $\{0\}$ respectively, it follows from the restricted convergence that we need only show that for any compact subset $E \subset \Omega_{\Gamma}$,

$$
\sup \left\{P_{\Gamma}\left(X_{0}, Y_{0}+Y\right): X_{0}+i Y_{0} \in E\right\} \leq k_{1}(Y)
$$


for some rectangular majorant $k_{1}$ satisfying 1.11 , and such that $\lambda^{n}\left(R_{j}\right) \geq \epsilon_{0}>0$ $\forall j$.

This is well known for the ordinary Poisson kernel, so we are left with (b). That problem clearly reduces to showing that $m_{\Gamma}^{2}(Y)$ is majorized by some such $k_{1}$, since multiples of $m_{\Gamma}^{2}$ majorize compact sets of translations of $m_{\Gamma}^{2}$.

Since any $\partial C_{Y^{\prime}}$ is rectifiable, if we define, for $0 \leq l \leq m$,

$$
S_{l, m}=\left\{Y:|Y| \leq 2^{m}, \operatorname{dist}(Y, \partial \Gamma U-\partial \Gamma) \leq 2^{l}\right\},
$$

there exists $N_{8}$ independent of $l, m$ and a finite family $R_{l, m}=\left\{R_{l, m, j}: 1 \leq j \leq\right.$ $\left.L_{l, m}\right\}$ of rectangles centered at 0 , such that $\lambda^{n}\left(R_{l, m, j}\right) \geq 2^{n+m+(n-1) l}, s_{l, m} \subset$ $\bigcup_{j}^{\prime, m} R_{l, m, j}$, and $\Sigma_{j} \lambda^{n}\left(R_{l, m, j}\right) \leq N_{8} \lambda^{n}\left(S_{l, m}\right)$. It is clearly possible to majorize $m_{\Gamma}^{2}$ by an $L^{1}$ sum of scalar multiples of characteristic functions of the $S_{l, m}$, and the proof that this sum satisfies the conditions for a rectangular majorant is straightforward.

3.8 Corollary. For every polytopic cone, the conclusion of 3.7 bolds.

Proof. We apply 1.16(d). The only detail that is not obvious is the hypotheses of 1.15 applying to all the $\Gamma_{i}$ in the special intersection with $T=T_{1}(\Gamma, P)$. Clearly, though, by $(15), T_{1}\left(\Gamma_{i}, P\right) \supset T_{1}(\Gamma, P)$ for each $i$; using the product expression for the Poisson kernel of an octant, the rest is straightforward.

Using 1.6, 1.16, and 2.10ff, one can construct other cones of more general shape in which the results of 3.7 hold. For instance, the cone with cylindrical section $\Gamma=(0, \infty) C^{0} \subset \mathbf{R}^{4}, C^{0}=\left\{(1, x, y, z): x^{2}+y^{2}<1,|z|<1\right\}$, is regular, and since it is the special intersection of two cones which are linear transforms of $\Gamma_{c} \oplus(0, \infty), \Gamma_{c} \subset \mathbf{R}^{3}$ the circular cone, it can also be shown to satisfy the conclusions of 3.7 whenever $r(\Gamma, P)=0$.

\section{BIBLIOGRAPHY}

1. S. Bochner, Group invariance of Cauchy's formula in several variables, Ann. of Math. (2) 45 (1944), 686-707. MR 6, 123.

2. L. J. Dickson, Some limit properties of.Poisson integrals and holomorphic functions on tube domains, Dissertation, Princeton University, Princeton, N. J., 1971.

3. G. H. Hardy and J. E. Littlewood, A maximal theorem with function-theoretic applications, Acta Math. 54 (1930), 81-116.

4. S. Karlin and L. S. Shapley, Geometry of moment spaces, Mem. Amer. Math. Soc. No. 12 (1953). MR 15, 512.

5. A. Korányi, The Poisson integral for generalized half-planes and bounded symmetric domains, Ann. of Math. (2) 82 (1965), 332-350. MR 34 \#371. 
6. E. M. Stein, G. Weiss and M. Weiss, $H^{p}$ classes of holomorphic functions in tube domains, Proc. Nat. Acad. Sci. U. S. A. 60 (1968), 1160-1162.

7. E. M. Stein and N. J. Weiss, On the convergence of Poisson integrals, Trans. Amer. Math. Soc. 140 (1969), 35-54. MR $39 \# 3024$.

DEPAR TMENT OF MATHEMATICS, UNIVERSITY OF WASHINGTON, SEATTLE, WASHING TON 98177

Current address: Department of Mathematics, University of New South Wales, Kensington 2033, Australia 